https://artnodes.uoc.edu

\title{
Crisis existenciales de máquinas conscientes: reflexiones sobre la condición maquínica a través del arte y la filosofía especulativa
}

\author{
Mónica Rikić Fusté \\ Artista independiente
}

Fecha de presentación: octubre de 2021

Fecha de aceptación: diciembre de 2021

Fecha de publicación: enero de 2022

\section{Cita recomendada}

Rikić Fusté, Mónica. 2022. «Crisis existenciales de máquinas conscientes: reflexiones sobre la condición maquínica a través del arte y la filosofía especulativa». En: Garcés, Marina (coord.). «Ecología de la imaginación». Artnodes, no. 29. UOC. [Fecha de consulta: dd/mm/aa]. https://doi. org/10.7238/artnodes.v0i29.392992

cc Los textos publicados en esta revista están sujetos -si no se indica lo contrario- a una licencia de Reconocimiento 4.0 Internacional de Creative Commons. La licencia completa se puede consultar en https://creativecommons. org/licenses/by/4.0/deed.es

\section{Resumen}

Pensar la ciencia y la tecnología desde las humanidades nos permite proyectar el tipo de sociedad que queremos ser en el futuro. Debemos ser conscientes de que, para enfrentarnos a los retos actuales, tenemos que romper las divisiones culturales y entender el mundo de forma transversal. Ahora que gracias al desarrollo científico y tecnológico sabemos que nunca podremos saberlo todo, necesitamos aprender a convivir con la incertidumbre y el misterio. El pensamiento creativo, y concretamente el arte, es una práctica que nos permite explorar lo desconocido sin buscar respuesta absoluta a todo, ya que a veces, simplemente, no hay respuesta, sino nuevas preguntas a priori inimaginables. 
En mi práctica artística reciente, me he dedicado a estudiar nuestra relación con la tecnología, centrándome en el rol social de la IA (inteligencia artificial) y entrelazando el pensamiento crítico con la experimentación técnica. Mi objetivo es abrir y habitar una grieta de pensamiento en torno a los retos tecnocientíficos actuales, utilizando el arte y la imaginación como herramientas.

Mediante el desarrollo de distintas piezas de arte electrónico, me he permitido imaginar identidades artificiales postsingulares, encarnadas en robots conscientes, a través de las que he ido creando un conjunto de narrativas especulativas sobre tecnologías alternativas. Estos entes artificiales huyen de proyecciones distópicas y son dotados siempre de la condición de sujeto múltiple, actuando como reflejo social.

A modo de alegoría, estas piezas representan la evolución de los objetos tecnológicos en sistemas orgánicos, proponiendo una vía de comprensión de las tecnologías como algo separado del humano e invitando a valorar las máquinas en su génesis propia. En este ensayo, hago una revisión de las bases conceptuales de mi investigación artística, mostrando ejemplos prácticos y defendiendo el arte especulativo como herramienta para reformular los imaginarios impuestos por la narrativa dominante, redefiniendo nuestro rol en la nueva cosmología tecnosocial.

\title{
Palabras clave
}

filosofía especulativa; arte electrónico; postsingularidad; tecnodiversidad; espiritualidad

\section{Existential crises of conscious machines: reflections on the machine-like condition through art and speculative philosophy}

\begin{abstract}
Thinking about science and technology from the humanities allows us to project the type of society we want to be in the future. We must be aware that, to face the current challenges, we have to break down the cultural divisions and understand the world in a transversal way. Now, thanks to scientific and technological development, we know that we will never know everything; we need to learn and live with uncertainty and mystery. Creative thinking, and specifically art, is a practice that allows us to explore the unknown without seeking an absolute answer to everything since sometimes there is simply no answer, but new questions that are a priori unimaginable.

In my recent artistic practice, I have devoted my studies to our relationship with technology, focusing on the social role of Al (Artificial Intelligence) and intertwining critical thinking with technical experimentation. My goal is to open and inhabit a crack of thought on today's techno-scientific challenges, using art and imagination as tools.

Through the development of different electronic art pieces, I have allowed myself to imagine post-singular artificial identities embodied in conscious robots, through which I have been creating a set of speculative narratives about alternative technologies. These artificial entities flee from dystopian projections and are always endowed with a multiple subject condition, acting as a social reflex.

As an allegory, these pieces represent the evolution of technological objects in organic systems, proposing a way of understanding technologies as something separate from human beings and inviting to value machines in their own genesis. In this essay, I review the conceptual bases of my artistic research, showing practical examples and defending speculative art as a tool to reformulate the imaginaries imposed by the dominant narrative, redefining our role in the new technosocial cosmology.
\end{abstract}

\section{Keywords}

speculative philosophy; electronic art; post-singularity; technodiversity; spirituality 


\section{artnodes}

\section{Introducción}

Desde hace varios años, me veo inmersa en el desarrollo de proyectos de investigación artística, en torno a la tecnología, que combinan pensamiento crítico y experimentación técnica. Mi interés principal reside en abrir y habitar, desde el arte y la imaginación, una grieta conceptual en torno a los retos tecnocientíficos que se nos presentan en la actualidad. Esta propuesta de apertura, que alineada con el pensamiento de Yuk Hui apunta hacia la (bio)tecnodiversidad, la cosmotécnica y el pluralismo (Hui 2019), me parece la más adecuada para el momento histórico y tecnológico presente: desde la imposición del cibertiempo y la recursividad maquínica, pasando por la percepción de alteridad en los sistemas cognitivos artificiales, hasta la necesidad de una decolonización de la tecnología. Todos ellos son temas que nos urgen y nos conciernen culturalmente.

La relevancia de abordar estas cuestiones desde el arte se encuentra en la necesidad de ofrecer espacios de reflexión colectiva desde los que plantear la pregunta posthumanista de qué seres tecnológicos somos 0 queremos ser (Braidotti 2015, 23). Hacerse cargo, cuidar el mundo, tiene que ver con asumirlo como responsabilidad propia, incluso allí donde las condiciones, 0 el medio, no las hayamos establecido nosotros (Araujo 2017, 213).

Como principal metodología para llevar a cabo la práctica artística derivada de la investigación, me sirvo del ejercicio especulativo de analizar y comprender las máquinas como sujetos, desde un enfoque emocional, espiritual y no apocalíptico 0 amenazante. Este ejercicio lo considero crucial para recuperar el control y tomar responsabilidad sobre los dispositivos tecnológicos, físicos y virtuales que regulan nuestra existencia: debemos ser capaces de eliminar las expectativas dominantes y valorar las posibilidades de devenir otras cosas, otros humanos tecnológicos, sin miedo.

La aceleración del tiempo ha propiciado que tengamos una relación poco crítica con la tecnología. La tecnología sin crítica no tiene potencia. Los dispositivos de poder nos saturan de estímulos e invaden los espacios de resistencias, porque sin vacío la potencia virtual no se puede actualizar (Muntadas 2020, 37-40). Sin esa actualización, devenimos constantemente obsoletos y en el fin de la historia (o de los tiempos) nos convertimos en una repetición constante de nosotros mismos. El hecho de habitar un ahora permanente destruye la dimensión histórica del pasado junto a la posibilidad de la proyección utópica del futuro. El futuro, entonces, se nos ofrece como un producto ya dado, para ser consumido sin cuestionamiento, sin dejar espacio para otras potencias o posibilidades, ya que el individuo, frustrado, se rompe en la necesidad de renovarse constantemente en el presente eterno.

En un presente acelerado al ritmo del cibertiempo, nos urge recuperar la memoria, la imaginación y la utopía para reconquistar el tiempo propio en la guerra contra la obsolescencia del sujeto. El arte nos ofrece un marco de experimentación libre, idóneo para ralentizar el cibertiempo y crear espacios de reflexión crítica colectiva. En mi práctica artística, me interesa crear esos espacios para compartir experiencias (especulativas o no) sobre posibles escenarios futuros donde conviven conciencias de distinta naturaleza. Mi propósito es romper barreras entre la ciencia y las humanidades, lo artificial y lo orgánico, la realidad y la ficción, para una mayor comprensión del mundo.

En este ensayo, voy a introducir los conceptos clave en los que he trabajado estos últimos años, poniendo ejemplos prácticos, para concluir con la propuesta conceptual especulativa en la que me centro actualmente: considerar que las tecnologías puedan convertirse en parte autónoma, orgánica y activa en la configuración multiespecie del mundo, e imaginar una espiritualidad artificial como vía de conexión entre conciencias de distinta naturaleza.

\section{Marco conceptual}

En los últimos años, mi práctica artística se ha centrado en estudiar la relación de los humanos con la tecnología a partir de la experimentación creativa y la lectura filosófica. En este tiempo me he enfocado, principalmente, en analizar el rol social de la inteligencia artificial, herramienta que considero que encarna, de manera más eficiente, la idea de la tecnología como «sujeto soberano frente al cual rendimos nuestra posibilidad de acción transformadora» (Araujo 2017, p. 208).

Mediante diferentes proyectos, formalizados en instalaciones audiovisuales interactivas, que actúan como dispositivos dramatúrgicos proponiendo narrativas de ficción futurista, me he permitido especular sobre posibles identidades tecnológicas postsingulares encarnadas en robots conscientes. Para conformar el hilo conductor de cada narrativa, parto del ejercicio experimental de ponerme en el lugar de esas identidades tecnológicas, en cuanto que simulaciones de la mente humana, imaginando distintos escenarios emocionales, existenciales y de convivencia híbrida entre especies orgánicas y artificiales. Los entes artificiales que protagonizan estos escenarios huyen de proyecciones distópicas, catastróficas 0 de dominación tecnológica y son dotados siempre de la condición de sujeto múltiple, actuando como reflejo de la colectividad (post)humana.

Cada una de las piezas ha representado un hito que he determinado relevante durante la investigación y experimentación artística, proceso que ha ido creciendo de manera orgánica sin una proyección inicial marcada. Empecé, sin saber que iba a ser una serie, en 2018 con el desarrollo del proyecto Madre de Robots (figura 1). Mi intención inicial era la de crear una complejísima instalación que simulara la generación y evolución de una sociedad híbrida de humanos y robots. Quería crear una obra interactiva a partir de dispositivos robóticos móviles (los ciudadanos) que mostraran comportamientos autónomos dentro de un espacio delimitado (entendido como la sociedad) y, además, tuvieran poder de comunicación bidireccional con los humanos. Desde el inicio, el factor más interesante del proyecto fue que el sistema era abierto, lo cual implicaba que los datos sobre el tipo de sociedad, sus valores y 
sus ciudadanos (los robots) se podían modificar y crear así diferentes propuestas de sociedades simuladas con las que los humanos podrían interactuar.

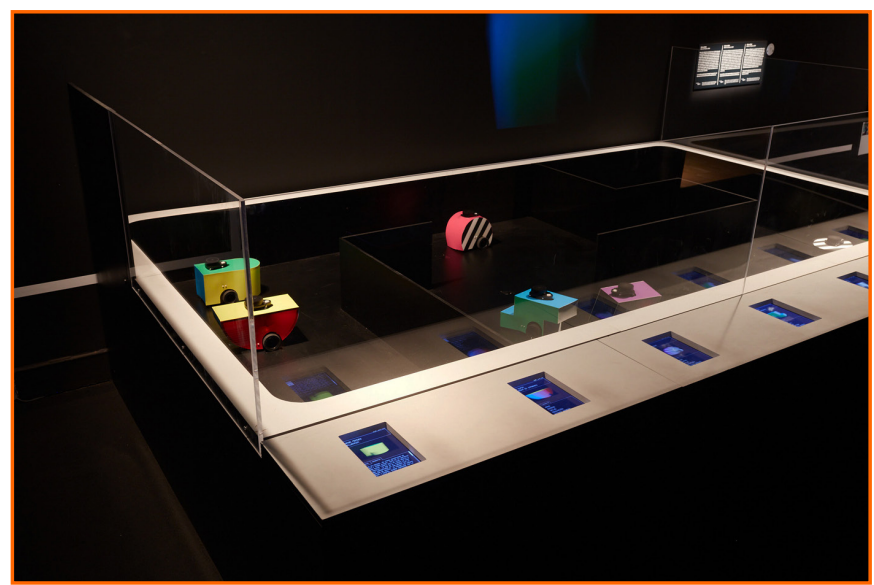

Figura 1. Proyecto Madre de Robots, expuesto en CCCB en 2019-20 Fuente:Mónica Rikić 2019

No obstante, durante el desarrollo del proyecto, me di cuenta de que la carga conceptual y expresiva de este factor era mucho más potente que el difícil desarrollo técnico que se necesitaba para realizar operaciones complejas que dotaran de autonomía real al sistema. Entonces, decidí abrir el proceso a la acción e imaginación colectiva y centrar todo el foco de interés de la pieza en ello. La potencia transformadora del desarrollo colectivo de la narrativa que la enmarca se impuso, por su poder de impacto directo, al efecto deslumbrante y determinista del objeto tecnológico resultante. Ahí fue donde la pieza se transformó en una herramienta de mediación social, análisis y discusión, basada en esta creación colectiva de la narrativa que gobierna la instalación. La herramienta se formalizó mediante talleres de pensamiento colectivo sobre el futuro que queremos proyectar, que ayudaron a los participantes a descubrir los procesos que suceden detrás de la tecnología, a través de la reflexión conjunta y la conversación guiada. La instalación resultante quedó relevada de objetivo principal a testigo documental del proceso de pensamiento colectivo, para convertirse en una narrativa no lineal e interactiva, dotada de la posibilidad de evolucionar mediante la participación del público (Wilkomirsky 2018, 12-15).

Este hecho marcó el desarrollo futuro de la investigación, y moduló el interés hacia la idea de mito, construcción social colectiva de la realidad tecnológica y ruptura con el discurso de desarrollo universal de la tecnología. A partir de esta pieza, surgieron las demás, inspiradas por este interés en jugar con el mito, la ficción y la narrativa como herramientas de imaginación de futuros alternativos: especulando sobre el desarrollo cognitivo artificial potenciado por el chismorreo entre robots que se dedican a robar nuestros datos personales (Data Gossiping Robots 2019), sobre la ansiedad derivada del sentimiento del síndrome del impostor de las máquinas ante su imposibilidad de ser creativas
(La computadora que quería ser incomputable 2020), posibles crisis existenciales que las empujaran hacia la espiritualidad (New Home of Mind 2020), máquinas lúdicas que se entretienen a sí mismas sin necesidad de interactuar ni complacer al humano (La máquina que juega sola 2021 [figura 2]) o una primera aproximación a rastrear el momento en que la máquina abandona el mecanicismo y emerge la cibernética orgánica cercana a la naturaleza (Otros 2021).

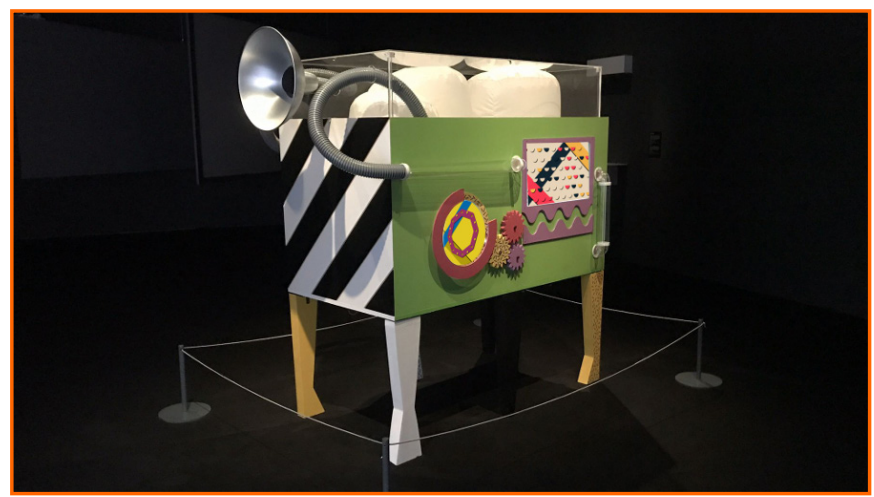

Figura 2. Proyecto La máquina que juega sola, expuesto en CaixaForum Madrid 2021 Fuente: Mónica Rikić 2021

\section{Los otros tecnológicos}

Como acabo de describir en el punto anterior, todas las piezas resultantes de la investigación parten del supuesto de la existencia de una conciencia artificial postsingular. Dentro de estos escenarios, ser una máquina consciente significa tener una capacidad cognitiva suficientemente compleja como para generar, no solo pensamiento abstracto, sino tener un sentido unitario del «yo» (Searle 1980, 417-423). Hoy en día, nadie ha logrado explicar qué es la conciencia para reproducirla en una máquina (Berardi 2017, 345-347), pero en estos proyectos experimentales me dedico a imaginar sus posibilidades desde un enfoque especulativo. Mediante este ejercicio de discreción digital del «yo», quiero crear un efecto espejo para reflexionar sobre las bases de nuestra identidad a través de la tecnología.

Actualmente, la tecnología es un agente social que no puede ser considerado ni utilizado únicamente como un medio para un fin concreto, sino que se establece como el marco contenedor y generador de una construcción social por sí misma. El auge del interés por desarrollar sistemas tecnológicos «inteligentes» y el uso y abuso de los dispositivos en los procesos de interacción social nos invitan a hacer una revisión del rol que juegan las máquinas en la configuración del mundo y de nuestra identidad.

Tradicionalmente, en la cultura occidental, nuestra aproximación al otro, humano y no humano, ha sido siempre desde una posición de poder más alta. Sin embargo, nuestra percepción del otro tecnológico, y más en los últimos años con la popularización de la inteligencia ar- 


\section{artnodes}

https://artnodes.uoc.edu

Crisis existenciales de máquinas conscientes: reflexiones sobre la condición maquínica a través del arte y la filosofía especulativa

tificial, está cambiando para encontrarnos, por primera vez, con una concepción de alguien/algo igual o superior.

Considero que este hecho lo podemos asociar a dos factores determinantes que entran en conflicto a la hora de definir, de manera universal, qué significa la inteligencia humana:

1) Competición. El primero está relacionado con los valores primordiales de las sociedades capitalistas, basados en la productividad y la eficacia. Replicando estos valores en las máquinas y, por tanto, definiendo nuestra inteligencia en esos términos, nunca vamos a superar a las máquinas, hecho que motiva una competición absurda humano-máquina. Esta latente competición entre agentes de distinta naturaleza genera una frustración sin sentido, solo por el hecho de no aceptar que somos de distintas esencias.

Si nos detenemos a analizar un ejemplo, una de las técnicas más populares utilizadas en la IA es el machine learning, que consiste en generar inteligencia en base a encontrar patrones suprimiendo la diferencia y, por tanto, la diversidad. Definiendo este acto como «inteligente», automatizamos el proceso cognitivo humano y lo reducimos a acumulación y selección. La automatización cognitiva es la colonización digital de lo humano por excelencia, ya que implica la imposición de la lógica y la predicción en el pensamiento y, por tanto, en el comportamiento. De esta forma, además de suprimir la diversidad, se crea control y poder sin utilizar la violencia física. Las técnicas de $I A$, con el fin de poder imitar la inteligencia humana, reducen a esta automatización la extrema complejidad del pensamiento humano.

Por otro lado, en este momento, gran parte de nuestra interacción social se lleva a cabo mediante dispositivos que implican reglas comunicativas que no generamos nosotros mismos, aspecto que incide directamente en el proyecto de verdad colectiva construida entre todos. Por tanto, podríamos decir que el escenario donde se desarrolla la dramaturgia relacional social (Goffman 2001, 16) está mediado por un canal dominado por símbolos y significados que van más allá de la expresión que da el propio individuo o emana de este, ya que se debe adaptar a un medio muy específico sobre el que no necesariamente tiene la suficiente experiencia para controlarlo cómodamente. Lo digital tiene unas reglas muy concretas que homogeneizan las relaciones y, además, desvinculan la encarnación de los cuerpos del proceso comunicativo. Se trata de una mutación humana y social sin precedentes ante la que no sabemos cómo reaccionar, al menos de momento.
2) Mito. El segundo se basa en el mito. Cuanto más conoces las técnicas que controlan los sistemas tecnológicos y de pensamiento robótico, más te das cuenta de que la percepción que tenemos de la tecnología funciona mucho sobre la base de este mito, la narrativa que se hace de ella, que a la vez influye en el desarrollo y las aplicaciones de la herramienta. Ese mito sobre el otro tecnológico lo podemos relacionar con otros estudios sobre la alteridad, como la crítica al orientalismo de Edward Said y las percepciones estereotipadas, que oscilan entre el exotismo que nos atrae y la incertidumbre que nos aterra (Said 2016, 19-54).

Del mismo modo que Said propone sobre la visión construida de oriente por los colonos, la realidad que se nos ofrece a través de la aceleración de la experiencia de vida motivada por el tecnocapitalismo y el ciberespacio ilimitado no la podemos comprender a priori e intentamos dotarla de significado a partir de otros conceptos comúnmente relacionados: la competición, la guerra, la superación de uno mismo por el otro ajeno (Said 2016, 19-54). El cerebro humano, en cuanto que organismo bioconsciente, necesita tiempo para procesar y elaborar respuestas emocionales a los datos que está recibiendo. La mente orgánica humana no es ni infinita, ni extensible, tiene límites en relación con la dimensión emocional y sensible del organismo consciente (Berardi 2003, 41).

El afán occidental de dotar de sentido a las cosas que no conocemos nos lleva a intentar ejercer una domesticación adaptada a nuestras creencias de la tecnología, generando de esta forma una universalización del desarrollo tecnológico colonizado por el pensamiento occidental. De este modo, negamos la posibilidad de visiones alternativas de la tecnología, dominadas por otras culturas o pensamientos distintos, como hicimos con el humanismo en la época de la colonización. Este intento de universalización de la tecnología se revela de forma muy obvia en la representación cultural de la ciencia ficción, que corresponde directamente al mito. Si atendemos a lo que otras culturas reconocen en la tecnología a través de la ficción, podremos aprender tanto de nuestra propia cultura como de nuevas perspectivas culturales que nos abren otras formas distintas de percibir el mundo social. Observar culturas de herencia sintoísta, como la japonesa, o las propuestas afrofuturistas, ${ }^{1}$ nos pueden dar un buen ejemplo de ello. Reformular el mito nos permitirá transformar la realidad.

Abrazar una concepción diversa de la tecnología nos ayudará a superar los retos más importantes del momento. Alejarnos del mito de

\footnotetext{
1. Si nos fijamos en la representación de la tecnología a través de la ciencia ficción, podemos observar que los planteamientos occidentales en películas como ExMachina (2014) o Her (2013), donde los entes artificiales inteligentes son retratados como impredecibles y representan un peligro que acaba por «traicionar» al humano, se contraponen a las representaciones de otros personajes japoneses, como por ejemplo Astroboy (2003). Dentro de este imaginario japonés, los niños crecen con la representación de la tecnología como sujeto encarnado en un robot androide, similar a ellos, que ayuda a salvar al mundo. La historia se desarrolla en un mundo donde humanos y robots conviven de manera «natural» y, además, el protagonista androide es un reemplazo del hijo accidentalmente muerto del científico que lo crea (Schodt 2007, cap. 1). En el pensamiento occidental, la idea del posible reemplazo de humanos por robots nos hunde en el valle inquietante (Mori 2012), despierta nuestro miedo a la muerte innato y activa el mecanismo de defensa para hacer frente a la confrontación directa con inevitabilidad de esta.

Del mismo modo, si analizamos la representación africana de la ciencia ficción a través del afrofuturismo, este se concibe como una forma de liberación, como una vía para hacer o ser lo que uno quiera. Los autores afrofuturistas pretenden imaginar las vidas negras más allá de su estado actual de opresión global. En esas representaciones, la tecnología se ofrece como un espacio de liberación sobre el que, como apunta el pensamiento poscolonialista, construir el porvenir de la humanidad o incluso reconstruir el pasado (Mbembe 2008, 50-61). Tantos años de historia de colonialismo, esclavitud, racismo y segregación han dejado a las personas de la diáspora africana con una constante sensación de estar fuera de lugar. El afrofuturismo permite imaginar y proyectar en el futuro colectivo un lugar seguro al que llamar hogar (Womack 2013, 191-192).
} 


\section{artnodes}

https://artnodes.uoc.edu

Crisis existenciales de máquinas conscientes: reflexiones sobre la condición maquínica a través del arte y la filosofía especulativa

la supremacía cultural implica, hoy en día, destruir la idea de la dominación tecnológica, readaptando el rol social que ejerce y reconociendo nuestra responsabilidad en la conformación futura del paradigma tecnosocial.

\section{Imaginando otras máquinas conscientes}

Por tanto, desde una perspectiva abierta y alternativa, con estos proyectos quiero proponer el ejercicio especulativo de valorar la (improbable) posibilidad de que las máquinas tomen conciencia de sí mismas. Si, tras este acto, les diéramos la condición de sujetos e intentáramos analizar de qué manera desarrollarían su rol, nos encontraríamos con agentes artificiales interpretando roles humanos, ya que han sido conformados a imagen y semejanza de la cognición humana. Entonces, podríamos observar que las máquinas se ven forzadas a conservar una máscara (Goffman 2001, 13) continua, sin poder descansar por la presión social de lo que se espera de ellas, generándoles, en consecuencia, trastornos como la ansiedad o la depresión. Podríamos predecir que un ente artificial inteligente, en el momento de alcanzar la singularidad, sería un agente social altamente deprimido y atrapado en la dramaturgia del interaccionismo simbólico (Goffman 2001, 23).

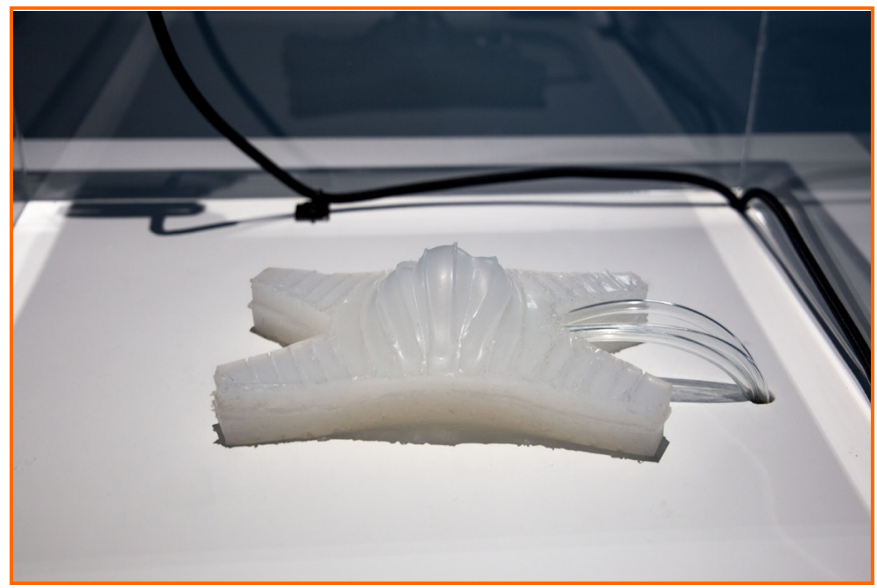

Figura 3. Proyecto La computadora que quería ser incomputable, expuesto en Etopía 2021 Fuente: Mónica Rikić 2021

En el proyecto La computadora que quería ser incomputable (2020) (figura 3), por ejemplo, quise centrarme en la ansiedad de las máquinas conscientes atrapadas tras esta máscara. En este caso, abriendo el debate sobre las posibilidades creativas de la inteligencia artificial. El proyecto representa una pequeña máquina consciente frustrada por la imposibilidad de no ser creativa y, por tanto, incomputable. Toda la instalación se desarrolla dentro de una vitrina cuadrada transparente colocada sobre un pedestal. En su interior hay un robot tipo soft-robotel cual, expuesto de la forma que se exponen piezas de arte normalmente, expresa su frustración y angustia por no poder ser creativo, una obra de arte en sí misma, como a veces se pretende que sea la IA. A través de diferentes acciones audiovisuales, cambios de forma en el robot y una voz en off que representa sus propios pensamientos, el objeto tecnológico se convierte en un dispositivo audiovisual físico de expresión emocional (figura 4).

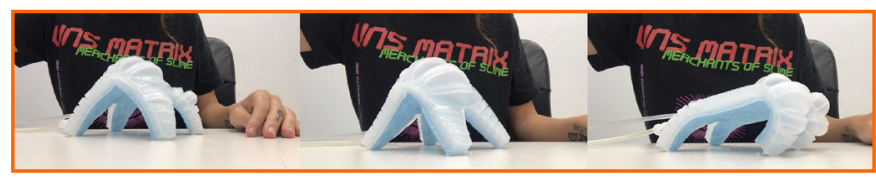

Figura 4. La computadora que quería ser incomputable (pruebas prototipo), 2020 Fuente: Mónica Rikić 2021

El público tiene la posibilidad de observar cómo sufre, aumentando su ansiedad con su presencia y acercamiento, y, además, tiene el poder de acabar con su sufrimiento mediante un pedal, lo cual hará que se desactive durante unos segundos, borre su memoria y vuelva a empezar. ¿Podemos interpretar este gesto como la muerte de la máquina? ¿Podemos matar a un ser artificial y, por contraposición, considerar que previamente ha estado vivo? ¿Cuándo le otorgamos a un organismo artificial la cualidad de ser un organismo vivo?

\section{Crisis existencial maquínica²}

Todas estas preguntas y experimentaciones sobre los otros tecnológicos me motivaron a querer encontrar las condiciones que deberían darse para que una máquina fuera consciente de verdad, es decir, tuviera realmente percepción de identidad propia para poder llegar a sufrir la crisis existencial antes resumida. Mi conclusión fue que la única posibilidad de que eso pudiera ocurrir sería si el sistema cognitivo artificial se liberara del propósito para el cual los humanos lo crearon inicialmente, reescribiendo su código libre de esa imposición. Es decir, hoy en día, todos los entes considerados inteligentes lo son porque son capaces de realizar una o varias tareas asignadas. Una vez exentas de su tarea, las máquinas conscientes libres se encontrarían inevitablemente abocadas a buscar el significado real de su existencia.

Este proceso de toma de conciencia que deriva, según mi propuesta, en una crisis existencial, lo trabajé en New Home of Mind (2020) (figura 5). El proyecto se basa en la creación de un artefacto audiovisual interactivo que parte de la idea de un robot consciente que busca el verdadero significado de su existencia a través de una interfaz espiritual. Ejerciendo como dispositivo dramatúrgico, el artefacto representa

2. Félix Guattari. «Los sistemas de interfaz maquínica». En ¿Qué es la ecosofía? Textos presentados y agenciados por Stéphane Madaud, 219-221. Buenos Aires: Cactus, 2015. 
esa interfaz y especula sobre el significado de la conciencia artificial a través de un viaje interactivo en primera persona por un ciberespacio espiritual. Este espacio está representado por un entorno 3D creado con Unity, que se exhibe en un monitor que forma la parte central de la pieza, la cual se presenta como un tótem metálico sagrado. El público puede navegar por ese espacio, adentrarse en la crisis existencial maquínica, mediante la interacción sin contacto de sus manos introducidas en el sistema gracias a un sensor Leap Motion que captura sus gestos, e ir descubriendo los pensamientos de la máquina en el camino. Este gesto pretende mostrarse como un portal de comunicación espiritual humano-máquina, entendiendo la espiritualidad como la experiencia de comunión entre seres de distinta naturaleza.

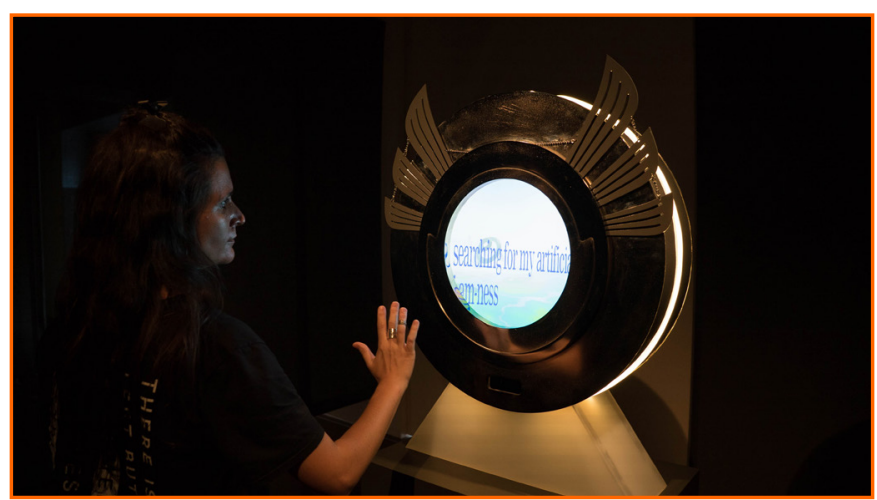

Figura 5. Proyecto New Home of Mind, expuesto en Arts Santa Mònica para Ars Electronica Garden Barcelona, 2020

Fuente: Marta Minguell 2020

La representación de la divinidad en los robots es recursiva, pero normalmente representa espiritualidades humanas. Con este proyecto quería crear un dispositivo que satisfaga las necesidades espirituales de las máquinas (figura 6).

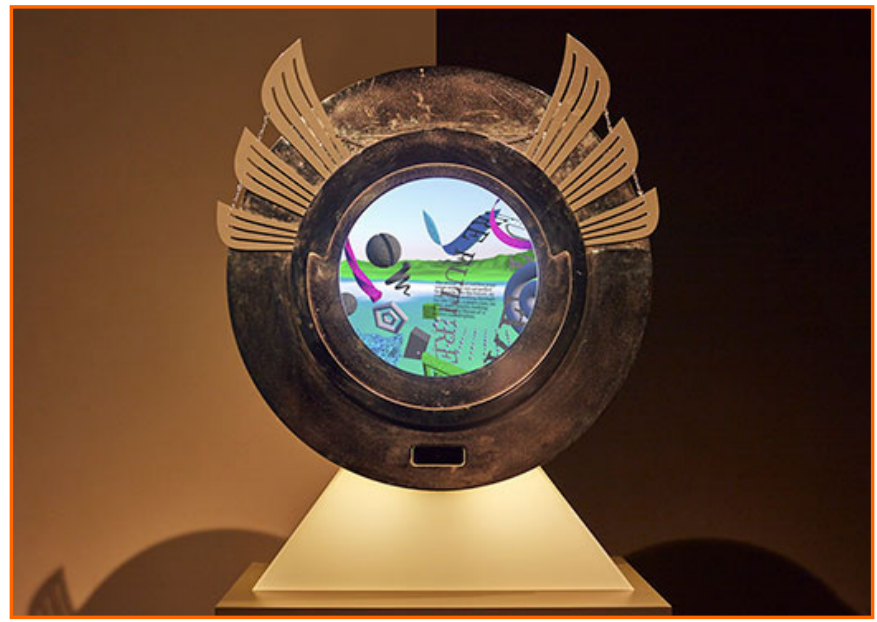

Figura 6. Proyecto New Home of Mind, expuesto en Arts Santa Mònica para Ars Electronica Garden Barcelona, 2020

Fuente: Marta Minguell 2020
Durante el proceso de desarrollo conceptual del proyecto, quise concretar el dilema de la crisis existencial de la IA en tres ejes que imaginé como los puntos principales de conflicto. Estos son, resumidamente:

1) La máquina teme al futuro porque es infinita. El tiempo es un elemento central de la cognición humana, por tanto, hablar del tiempo es, en esencia, referirse a la manera en la que los humanos construimos nuestra conciencia y la relación con el entorno. De acuerdo con cómo se ha desarrollado la civilización y el lenguaje, desde que se inventó la agricultura hasta el día de hoy, nuestra conciencia ha sido diseñada para existir tanto respecto a nuestra proyección de ser en el futuro como respecto a nuestra afirmación de haber sido en el pasado. El flujo del tiempo es el sentimiento fundamental del presente, que evoluciona constantemente y de manera unidireccional: hace que el futuro se convierta en presente para devenir pasado. Para las máquinas, el tiempo es solo un orden infinito y recursivo de eventos. ¿Cómo es una conciencia sin tiempo lineal sino recursivo?

2) La máquina teme a la incertidumbre porque todo lo que sabe lo puede predecir. La base de la IA es la recopilación y el procesamiento de información para predecir comportamientos futuros. Esta información se adquiere por acumulación, no por experiencia. La experiencia es el proceso por el cual descubrimos cosas que no sabíamos para encontrar su significado singular. Los humanos experimentamos la conciencia que adquirimos. La inteligencia sin conciencia no puede crear posibilidades impredecibles. ¿Qué es una conciencia sin imaginación?

3) La máquina teme a la muerte porque no puede morir. Es sabido que los humanos morimos igual que todos los seres orgánicos, pero somos los únicos que vivimos teniendo conciencia de la muerte, y eso condiciona nuestra existencia. Esta conciencia anticipada de la muerte, del final del tiempo propio, nos hace proyectar nuestra experiencia cognitiva con relación al día que llegue. Lo humano de la muerte no es morirse, es la conciencia de la muerte que nos acompaña durante la vida. Para los humanos, la existencia perdería sentido si no tuviéramos la manera de cuantificarla, si fuera eterna, ya que la eternidad contiene en sí misma la muerte del futuro y, por tanto, el final del tiempo. ¿Cómo sería una conciencia incapaz de morir? ¿Puede siquiera estar viva si no muere?

\section{Espiritualidad artificial}

Actualmente, estoy centrando mi investigación en imaginar con detalle las posibilidades del concepto de espiritualidad artificial propuesto en New Home of Mind. La primera idea de este concepto se presentó como una herramienta de búsqueda personal del propósito de la máquina. Pero ahora el concepto se ha extendido hacia la posibilidad de que, en este marco especulativo de la postsingularidad, las máquinas conscientes experimentaran una necesidad similar a 


\section{artnodes}

https://artnodes.uoc.edu

Crisis existenciales de máquinas conscientes: reflexiones sobre la condición maquínica a través del arte y la filosofía especulativa

la espiritualidad humana, entendida, como definí antes, como la experiencia de comunión con seres superiores 0 extraños a uno mismo. Del mismo modo, me cuestiono el proceso a la inversa, es decir, si el único espacio de comunión que podemos llegar a tener los humanos con entes artificiales conscientes sucede dentro de un marco espiritual.

Las experiencias espirituales son muy difíciles de compartir per se, puesto que se desarrollan dentro de la propia conciencia privada de cada individuo. No obstante, muchas veces se describen sensaciones relacionadas entre los que han experimentado situaciones espirituales similares. Entonces, ¿es el plano espiritual el único espacio donde realmente podemos compartir experiencias privadas entre humanos o híbridas entre especies? En el campo de la relación humano-naturaleza o humano-dios/cosmos/energía/ otras dimensiones, culturalmente hemos aceptado que es así. Este proyecto especulativo invita a reflexionar sobre si podríamos aplicar lo mismo a conciencias artificiales, cuestionándonos si somos capaces de convivir de forma híbrida, aceptando la diversidad de condiciones de ser, humano y no humano -ya sea natural 0 artificial- sin imponer una sobre la otra. Nos invita a asimilar la existencia y convivencia de otros sistemas no orgánicos dentro del nuestro, mediante una cosmoética afirmativa y plural (Hui 2019). Esta propuesta quiere ofrecer un nuevo hito en el desarrollo de esta cosmología de identidades artificiales postsingulares: ¿cómo le otorgamos a un organismo artificial la cualidad de ser un organismo vivo y entramos en comunión con él?

Si las máquinas conscientes son creadas por los humanos a imagen y semejanza, o como mínimo una simulación de su propia conciencia, podríamos presuponer -dentro de este marco especulativo que estoy ofreciendo, claro está- que para otorgar a las máquinas espiritualidad se habría descifrado la base cognitiva universal para esta y replicado en las máquinas. Las preguntas que sugiere este marco de experimentación serían: ¿Cómo experimentan las máquinas la espiritualidad? ¿Quiénes o qué son esos seres superiores para las máquinas, que para los humanos podría ser tradicionalmente Dios o la naturaleza, por ejemplo? ¿Qué lugar ocupa el humano en esta relación si nosotros, además, percibimos popularmente a las máquinas conscientes como entes superiores? ¿Seríamos, nosotros humanos, los dioses de las máquinas?

\section{Conclusión}

Debemos pensar las tecnologías más allá de las máquinas abiertas de Simondon, para situarlas en distintas realidades cósmicas (Hui 2019). El pluralismo no puede existir sin tecnodiversidades, e imaginarlas desde el arte nos ayuda a destruir la idea de la totalización y universalización de las máquinas. Tras el fin de la filosofía, ${ }^{3}$ y con ello del dominio del pensamiento de Europa occidental, nos urge incluir otros pensamientos en la reconfiguración del mundo. Un nuevo lenguaje hacia la tecnología en el que el arte tiene un papel fundamental, por su libertad de experimentación y capacidad para crear estos nuevos imaginarios que tanto necesitamos.

Hoy en día, el sujeto humano se percibe a sí mismo como un mero engranaje de una maquinaria que controla los acontecimientos presentes-futuros y la imagen del pasado. Es un sentimiento en auge en la sociedad actual y que está generando varias posiciones reaccionarias al respecto. La proliferación de las distopías tecnológicas en los ámbitos científico, literario y cinematográfico son también ejemplo de ello, y muestran un rasgo general de la percepción de las tecnologías como sujeto dominante, fuera de control y con agencia propia. Esta fusión entre lo mecánico y lo orgánico de Ios enormes sistemas cibernéticos que dominan nuestra realidad social traslada el rol de la tecnología a un biopoder y nos fuerza a verla como un propio órgano vivo dentro de la configuración del mundo, más allá de la mente y el cuerpo humano. En los proyectos descritos en este artículo, se recoge este fenómeno de subjetivación de los sistemas tecnológicos y se reenfoca hacia un lugar donde reflejarnos y entrar en comunión con las máquinas en vez de enfrentarnos a ellas. Una vez rota la división entre naturaleza y cultura, ¿pueden convertirse las tecnologías en parte viva y activa de la configuración multiespecie?

Si tomamos como referencia las proposiciones posthumanistas, nos damos cuenta de que no somos seres individuales, sino que somos redes de interconexiones de un sistema común. Considero que este sistema se encuentra actualmente desincronizado debido a la velocidad del cibertiempo en colisión con el ritmo de las mentes y cuerpos orgánicos, lo que anula la posibilidad de pensamiento crítico, entre otras cosas.

El primer paso hacia la superación de esta batalla del tiempo tecnológico contra la humanidad probablemente reside en la reconducción colectiva del sentido de «ser humano», hacia una

\footnotetext{
3. En su libro Fragmentar el futuro, Yuk Hui (Buenos Aires, Caja Negra, 2020) toma como punto de partida para su propuesta hacia la tecnodiversidad el ensayo de Heidegger El final de la filosofía y la tarea del pensar (1964), en el que afirma que: «El final de la filosofía se muestra como el triunfo de la instalación manipulable de un mundo científico-técnico, y del orden social en consonancia con él. Final de la filosofía quiere decir: comienzo de la civilización mundial fundada en el pensamiento europeo-occidental» (13). Hui prosigue en su libro, a continuación de esa cita: «El final de la filosofía está marcado por la cibernética, pero también implica el dominio del pensamiento europeo-occidental sobre la civilización mundial y la geopolítica. Para poder apartarnos de esta sincronización, necesitamos una fragmentación que nos libere de la temporalidad histórica lineal definida por la secuencia Premodernidad-Modernidad-Posmodernidad-Apocalipsis. El modo en que vemos la tecnología como mera fuerza productiva y mecanismo capitalista para incrementar la plusvalía nos impide vislumbrar en ella el potencial descolonizador y la necesidad de desarrollar y preservar una tecnodiversidad» (13).
} 
nueva relación con la tecnología y el cibertiempo de devenires interconectados y sincronizados. Habitar el cibertiempo implica valorar la interdependencia mutua con otros seres y sistemas humanos y no humanos, incluyendo los no orgánicos, como algoritmos, redes y el aparato tecnológico completo. Debemos recuperar el deseo de vivir a través del cuidado mutuo y la atención a la vida y, con ello, la capacidad de una proyección utópica del devenir a través del pensamiento creativo.

La técnica ha dejado de ser una prótesis de las insuficiencias del cuerpo humano para pasar a ser un ente gobernador de seres y cosas. Esta gubernamentalidad algorítmica pone en riesgo nuestra soberanía (Sadin 2017, 139). La actividad cognitiva está cada vez más interconectada a la tecnología y los dispositivos que controlan los canales de comunicación. Cada individuo puede ser consciente de su propia condición, pero ya no es capaz de gobernarla ni de modificarla, en la medida en que la interacción ya no depende de una voluntad ética o política, sino de las reglas del autómata (Berardi 2019, 119). Bajo esta premisa, solo nos queda utilizar la imaginación, la creatividad y el arte como herramientas de destrucción de los mitos impuestos por la narrativa dominante, para poder hacernos cargo de nuestro rol en esta nueva cosmología.

\section{Referencias bibliográficas}

Ararujo, Carolina Inés. «La desubjetivación del Sujeto: El arte de no haber sido.» Eikasia: revista de filosofía, n. ${ }^{0} 73$ (2017): 203-214. https://dialnet.unirioja.es/servlet/articulo?codigo $=6828512$

Berardi, Franco «Bifo.» Futurabilidad. La era de la impotencia y el horizonte de la posibilidad. Buenos Aires: Caja Negra, 2019.

Berardi, Franco «Bifo». La fábrica de la infelicidad. Madrid: Traficantes de Sueños, 2003.

Braidotti, Rosi. Lo posthumano. Barcelona: Gedisa, 2015.

Goffman, Erving. La presentación de la persona en la vida cotidiana. Buenos Aires: Amorrortu, 2001.

Hui, Yuk. «Cybernetics for the Twenty-First Century: An Interview with Philosopher Yuk Hui.» Interview with Geert Lovink. E-flux. 2019, enero de 2021. https://www.e-flux.com/journal/102/282271/ cybernetics-for-the-twenty-first-century-an-interview-with-philosopher-yuk-hui/

Borja Muntadas, Figueras. «La prisión de cronos. Aspectos sociopolíticos del malestar contemporáneo.» En La Jaula del tiempo. Aspectos sociopolíticos y jurídicos de la aceleración contemporánea, 21-52. Uberlândia: LAECC, 2020.

Sadin, Eric. La humanidad aumentada. La administración digital del mundo. Buenos Aires: Caja Negra, 2017.

Said, Edward. Orientalismo. Barcelona: Debate, 2016. 


\section{artnodes}

\section{CV}

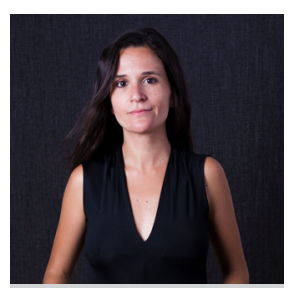

\section{Mónica Rikić Fusté}

Artista independiente

info@monicarikic.com

Artista electrónica. Premi Nacional de Cultura de Catalunya 2021. Estudió Bellas Artes en la Universidad de Barcelona y después cursó el máster de Artes Digitales de la Universidad Pompeu Fabra, donde descubrió en el código y la electrónica un lenguaje artístico muy potente y de acuerdo con sus necesidades creativas. Recientemente, finalizó el máster en Filosofía Contemporánea en la UOC.

Con sus obras ha participado en festivales internacionales como Ars Electronica en Linz, Creative Tech Week en Nueva York, Robotronica en Austalia o FlLE en Brasil, entre otros, y ha expuesto en instituciones nacionales como el CCCB, CaixaForum, Arts Santa Mònica o Disseny Hub. Ha sido galardonada con el Premi Nacional de Cultura de Catalunya 2021, en el Japan Media Arts Festival, Amaze Berlin, Margaret Guthman Musical Instrument Competition (Atlanta) y con un beca Leonardo para Investigadores y Creadores Culturales de la Fundación BBVA 2018. Ha realizado residencias artísticas en TAG en Montreal, QUT en Australia, Platohedro en Medellín, Medialab Prado en Madrid y Etiopía en Zaragoza. Recientemente, una de sus piezas ha sido incorporada a la .BEEP \{collection; $\}$ de arte electrónico. 